\title{
La incorporación de los académicos a la Universidad Veracruzana: socialización y estrategias de los agentes en las disciplinas
}

\author{
Eréndira García García' \\ José Luis Suárez Domínguez'
}

\section{RESUMEN}

El presente artículo, expone los resultados de un proyecto de investigación emprendido en 2016 y cuyo objetivo fue conocer las estrategias empleadas por los académicos para incorporarse a la vida universitaria. Los académicos son agentes que han construido un sentido práctico resultado de la socialización que tuvieron desde su posición como estudiantes en un determinado campo disciplinario. Se utiliza el método comparativo para conocer el modo en que cada disciplina construye su propia simbología, sus prácticas y sus reglas de juego, definidas como el marco de la formulación estratégica de los académicos.

PALABRAS CLAVE

agentes; académicos; incorporación; estrategias; disciplinas.

'Universidad Veracruzana, Veracruz, México. 


\section{THE INCORPORATION OF THE ACADEMICS TO THE} UNIVERSITY VERACRUZANA: SOCIALIZATION AND AGENT'S STRATEGIES IN THE DISCIPLINES

\section{ABSTRACT}

This article presents the results of a research project undertaken in 2016, whose objective was to know the strategies used by academics to join university life. Academics are agents who have constructed a practical sense as a result from the socialization they had from their position as students in a particular disciplinary field. The comparative method is used to know how each discipline constructs its own symbology, its practices and its rules of game defined as the framework of the academic's strategic formulation.

KEYWORDS

agents; academics; incorporation; strategies; disciplines.

\section{A INCORPORAÇÃO DOS ACADÊMICOS À UNIVERSIDADE VERACRUZANA: SOCIALIZAÇÃO E ESTRATÉGIAS DOS AGENTES NAS DISCIPLINAS}

\section{RESUMO}

Este artigo apresenta os resultados de um projeto de pesquisa realizado em 2016 cujo objetivo foi conhecer as estratégias empregadas pelos acadêmicos para se incorporar à vida universitária. Os acadêmicos são agentes que têm construído um sentido prático resultante da socialização que obtiveram de sua posição quando estudantes em um campo disciplinar particular. O método comparativo é usado para saber o modo como cada disciplina constrói a sua própria simbologia, suas práticas e suas regras de jogo, definidas como o arcabouço da formulação estratégica dos acadêmicos. 


\section{INTRODUCCIÓN Y ACERCAMIENTO A LA PERSPECTIVA TEÓRICA ADOPTADA}

Uno de los ámbitos constitutivos de las instituciones universitarias como organizaciones que giran alrededor del conocimiento es el de los académicos y los procesos que regulan su desempeño, sus trayectorias y la constitución de sus habitus como agentes pertenecientes al campo académico. En la sociología de Bourdieu (1995), las nociones de habitus y campo tienen gran utilidad para el estudio de los agentes académicos tanto como de las disciplinas en las que ellos se ubican.

La lógica de los campos se define como un espacio de luchas, como una red de relaciones objetivas en la que sus agentes desarrollan un sentido práctico. El babitus es un generador de disposiciones. En el caso del campo académico, el habitus es incorporado por los agentes académicos en la medida en que han construido una historia dentro de una institución específica (en nuestro caso de estudio dentro de la institución universitaria) y en el contexto de una disciplina en particular (Bourdieu, 2005).

El trabajo académico es inherente a las disciplinas y profesiones en las cuales tiene su base. Las disciplinas tienen tradiciones propias, ritos, costumbres y estilos (Becher, 1997; Bourdieu, 2005; Clark, 1991) que constituyen estructuras específicas bajo las cuales se organiza el trabajo académico. En el contexto de tales tradiciones pueden estudiarse diversos fenómenos, entre los que se cuenta el proceso de incorporación de los académicos a las comunidades disciplinarias, objeto de estudio constituido tanto en el plano internacional (Altbatch y Finkelstein, 1997) como en el nacional (Galáz et al., 2016; Gil Antón et al., 1994; Grediaga, Rodríguez y Padilla, 2004).

Si bien forma parte de la literatura actual, la incorporación de los académicos a las universidades es una zona de incertidumbre; es decir, un espacio dentro de la acción organizada que no tiene reglas de juego claramente establecidas (Crozier y Friedberg, 1990). Esto obedece a que las instituciones universitarias gozan de una autonomía relativa con respecto a esferas de poder más amplias, son entidades con su propio devenir histórico. El desarrollo y la evolución que ha tenido una institución apenas se equipara al de otras. Hay, entonces, fórmulas diversas en la incorporación de los académicos a la vida universitaria.

Una noción teórica útil para el estudio de la incorporación de los académicos es la de estrategia. Construida dentro de la misma perspectiva teórica asumida, la estrategia es al mismo tiempo resultado de las características del campo y de las disposiciones generadas por el habitus. Los académicos construyen estrategias de incorporación, tomando en cuenta los recursos que la universidad (institución que representa al campo) produce. Los académicos ponen en juego un sentido práctico que han incorporado en su paso por las instituciones. Las trayectorias que han derivado de las experiencias institucionales orientan los modos estratégicos. Las estrategias de los académicos son el resultado de una doble acción: la que ejerce el campo en ellos, y la que ellos construyen por pertenecer a un campo. 
En el caso de la Universidad Veracruzana (UV) no existe un estudio amplio y reciente sobre el fenómeno de incorporación de los académicos a las disciplinas. ${ }^{1}$ $\mathrm{Al}$ emprender un proyecto de esta naturaleza se buscó producir información relevante para conocer a estos agentes y crear un marco de referencia para el desarrollo de otras temáticas relacionadas.

Hasta ahora, los académicos son concebidos en la UV como conjuntos de cifras que conforman "los cortes de caja" para alimentar los anuarios estadísticos donde se describen rasgos muy generales sobre ellos. No es suficiente conocer a los académicos únicamente por el número que representan en la institución ni tampoco por el tipo de contratación o el aumento de plazas reportado en las últimas décadas. Es importante iniciar una discusión en la UV sobre el objeto en cuestión; tratar el tema con mayor complejidad, considerar la socialización de estos agentes, la construcción de sus trayectorias, sus expectativas, entre otros aspectos.

Para contar con una perspectiva adecuada para el estudio de los académicos y la forma en que ellos se integran a las comunidades disciplinarias en la UV, en este artículo se han considerado dos criterios. Primero, la forma en que las comunidades académicas y disciplinarias establecen reglas formales e informales para la aceptación de nuevos miembros. Segundo, las estrategias que los agentes académicos construyen para incorporarse a la vida académica. Ambas cuestiones están interconectadas.

Considerado lo anterior, cabe preguntarse, a manera de un planteamiento del problema de investigación, cuál es el grado en que las comunidades disciplinarias definen la participación e incorporación de nuevos miembros a sus territorios académicos, mediante qué mecanismos se expresa tal participación y qué procesos de selección están presentes. Pero al mismo tiempo puede plantearse qué hacen los agentes académicos que aspiran a ingresar a las instituciones, qué tipo de trayectorias construyen y qué escenarios de socialización los posicionan para construir esas aspiraciones.

Por la relevancia que tienen las comunidades disciplinarias en este estudio - pues alrededor de ellas gira tanto la idea de disciplina como la de organización - es pertinente hacer una distinción conceptual y empírica. Por disciplina se entenderá el cuerpo de conocimientos acumulados a lo largo del tiempo en torno a una serie de objetos de estudio delimitados a partir de un conjunto de métodos. Por organización o comunidad universitaria se hará alusión a las facultades, en tanto espacios encargados de recrear (impartir, transmitir) los conocimientos disciplinarios. Esta distinción sigue la lógica del clásico trabajo de Clark (1991) en el cual es posible observar los modos de autoridad que se desprenden de las disciplinas y de los establecimientos encargados de su recreación.

Para aproximar algunas respuestas al planteamiento formulado, se diseñaron algunas dimensiones de análisis que pueden aportar datos para cumplir tal propósito. Estas dimensiones están representadas por las políticas educativas, la producción simbólica y la socialización de los agentes académicos. Las tres dimensiones consti-

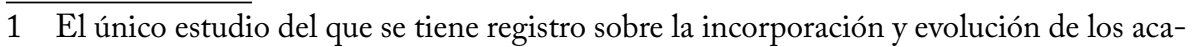
démicos en la UV fue elaborado a nivel nacional, el de Los rasgos de la diversidad, trabajo coordinado por Gil Antón y el Equipo Interinstitucional de Investigadores sobre los Académicos Mexicanos, en 1994. Este trabajo recuperó parcialmente datos de la UV y otras instituciones en el país. 
tuyen recursos analíticos para identificar los procesos de construcción de las estrategias de los académicos, lo cual da paso al fenómeno de incorporación ya enunciado.

\section{LAS ESTRATEGIAS METODOLÓGICAS EMPLEADAS}

El estudio fue realizado bajo una perspectiva metodológica de corte cuantitativo, haciendo uso de la comparación. En términos de su diseño, se optó originalmente por la recuperación de la información a partir de la encuesta ${ }^{2}$ a los académicos de nueve disciplinas ${ }^{3}$ aunque finalmente el estudio se concretó con únicamente cinco. El propósito fue comparar los procesos de incorporación y despejar los cuestionamientos formulados anteriormente. La selección de las disciplinas se hizo con base en tres criterios: su antigüedad como carrera en la UV; el tipo de disciplina; y finalmente, la edad de los académicos que conforman las poblaciones en cuestión.

Con respecto a la antigüedad de las carreras en las que se imparten las disciplinas, se planteó la introducción de varios contextos temporales; es decir, que en la historia de las carreras hubieran tenido lugar diversos procesos de reclutamiento para observar las "fórmulas" empleadas en cada contexto. Para el segundo criterio, el tipo de disciplina, se hizo referencia al modelo propuesto en Becher sobre la clasificación de las disciplinas universitarias. En este modelo las disciplinas se definen como blandas y duras en un cuadrante, y puras y aplicadas en otro cuadrante. El principio básico de tal clasificación disciplinaria consiste en que dentro de las sociedades de conocimiento hay una relación entre los aspectos sociales y las propiedades epistemológicas de dichas formas de conocimiento (Becher, 1997).

En el mismo orden de ideas, Becher define las combinaciones disciplinarias de la siguiente forma:

En las disciplinas duras puras, el conocimiento tiene un crecimiento acumulativo y sostenido. Hay una aparente claridad en los criterios para establecer o para refutar el nuevo conocimiento. Cuando una nueva conclusión es aceptada se le considera un descubrimiento y puede convertirse en un nuevo tipo de explicación.

En las disciplinas blandas puras, hay diversos criterios y una falta de consenso respecto de qué es un aporte auténtico en un campo en particular. Las explicaciones tienden a ser más débiles que en el conocimiento duro puro. Existe mayor permeabilidad en las zonas límites del conocimiento. El trabajo académico atraviesa a

2 La encuesta se diseñó con 37 preguntas distribuidas en las dimensiones de análisis y se aplicó en agosto de 2016. Algunas preguntas fueron recuperadas de otros instrumentos diseñados para el estudio de los académicos mexicanos, particularmente del trabajo La reconfiguración de la profesión académica en México (Galáz et al., 2012); y el cuestionario del "Estudio comparativo sobre el impacto disciplinario en las trayectorias académicas de los profesores de educación superior en México" (Grediaga, 2000); así como el instrumento empleado en el estudio Los rasgos de la diversidad (Gil Antón et al., 1994).

3 No obstante, en cuatro carreras no fue posible reunir un mínimo de casos, pues algunas veces los directores de las carreras no permitieron el acceso. En otras ocasiones no hubo disponibilidad de los académicos para contestar la encuesta. A menudo los académicos argumentaron el riesgo de proporcionar sus datos, a pesar de la confidencialidad garantizada por parte de los encuestadores. 
menudo terrenos ya explorados por otros. Las cuestiones básicas siguen siendo las mismas de una generación a la siguiente.

Por su parte, las disciplinas aplicadas también se clasifican en combinaciones que permiten identificar diferencias importantes. De esta forma, el conocimiento duro aplicado no es necesariamente acumulativo. Las disciplinas se dirigen típicamente hacia un fin práctico porque suelen centrarse en el control del mundo físico.

En cuanto a las disciplinas blandas aplicadas, se tiene que estas recurren al conocimiento blando puro como medio para comprender y aceptar la complejidad de las situaciones humanas. No son tan estables ni tienen un sentido de progresión tan eminente como el conocimiento duro aplicado. Sus funciones son juzgadas en términos pragmáticos y utilitarios.

La caracterización anterior constituye la base del segundo criterio establecido para la selección de las disciplinas bajo estudio. La pertenencia a las combinaciones explica por qué las disciplinas producen su propia simbología, así como sus referentes de identidad.

En cuanto a la edad de los académicos como tercer criterio de selección de las disciplinas, se buscó que las plantas académicas tuvieran cierta variabilidad para tipificar las estrategias empleadas por estos agentes. Como resultado de los criterios anteriores, se procedió a la elección de las cinco disciplinas. Estas cumplen las condiciones establecidas en el estudio (Cuadro 1).

Se seleccionó una disciplina dura pura (matemáticas), una dura aplicada (biología), dos blandas puras (sociología y lengua y literatura hispánicas), y una de corte blanda aplicada (pedagogía). Asimismo, la antigüedad de todas ellas permite ver diversos procesos de incorporación. Por su parte, la edad de los académicos presenta una variabilidad suficiente para cumplir con las disposiciones planteadas. La población encuestada alcanzó los 95 casos. No se intentó un muestreo, sino un censo; sin embargo, en algunas disciplinas se logró mayor cobertura que en otras. ${ }^{4}$

Cuadro 1 - Características de los académicos en las disciplinas bajo estudio.

\begin{tabular}{|c|c|c|c|c|c|c|c|}
\hline \multirow{2}{*}{ Facultad } & \multirow{2}{*}{$\mathrm{N}$} & \multicolumn{2}{|c|}{ Género } & \multirow{2}{*}{$\begin{array}{l}\text { Año de origen } \\
\text { de la carrera }\end{array}$} & \multirow{2}{*}{$\begin{array}{c}\text { Cobertura } \\
(\%)\end{array}$} & \multirow{2}{*}{$\begin{array}{c}\text { Edad } \\
\text { promedio }\end{array}$} & \multirow{2}{*}{$\begin{array}{l}\text { Rango } \\
\text { de edad }\end{array}$} \\
\hline & & $M$ & $\mathrm{~F}$ & & & & \\
\hline Biología & 14 & 10 & 4 & 1979 & 30 & 45 & $33-69$ \\
\hline Matemáticas & 15 & 13 & 2 & 1964 & 83 & 51 & $36-69$ \\
\hline Pedagogía & 26 & 10 & 16 & 1954 & 25.3 & 53 & $36-69$ \\
\hline Sociología & 20 & 13 & 7 & 1977 & 90 & 53 & $37-66$ \\
\hline Letras & 20 & 7 & 13 & 1956 & 80 & 43 & $28-70$ \\
\hline
\end{tabular}

$\mathrm{N}$ : número de participantes (total 95).

Fuente: base de datos de búsqueda.

Elaboración propia.

4 En los casos de pedagogía y biología los porcentajes de académicos encuestados fueron bajos. Sin embargo, dada la complejidad que implica encuestar a los académicos, se tomó la decisión de que ambas permanecieran en el estudio, pues logran cierta representatividad en su población. 


\section{FORMULACIÓN DE UNA HIPÓTESIS INICIAL}

A manera de hipótesis, se plantea que los procesos de incorporación de los académicos responden a las diferentes racionalidades establecidas por las disciplinas. Estas han construido históricamente sus propias dinámicas, resultantes de procesos internos y externos a su propio campo. Las disciplinas reciben una influencia por parte de las políticas públicas, las cuales han reorientado en las últimas décadas los procesos de incorporación de los académicos en la medida en que han promovido nuevos perfiles académicos en estos agentes. Asimismo, la incorporación se ve afectada por la producción simbólica y las condiciones de socialización experimentadas por los académicos antes del ingreso a la vida universitaria. Estas regulaciones dan lugar a un conjunto de estrategias construidas por los agentes académicos en función de los recursos de los que disponen, sin dejar de tomar en cuenta las características de las disciplinas mismas, su historia, sus procesos, sus códigos y su constitución como tales.

\section{LA OPERACIONALIZACIÓN DE LAS DIMENSIONES DE ANÁLISIS}

Las dimensiones de análisis fueron operacionalizadas para recuperar y analizar la información del trabajo empírico y explicar los procesos de incorporación de los académicos a la vida universitaria.

En el caso de la dimensión "políticas públicas", considerada como un conjunto de acciones que regulan de forma externa al campo universitario - y de manera específica a las disciplinas en su ámbito inmediato -, hay una relación con el fenómeno de la incorporación porque mediante sus disposiciones (obtención de grados académicos, diversificación del trabajo académico, pertenencia al Sistema Nacional de Investigadores (SNI) y al Programa para el Desarrollo Profesional Docente (PRODEP), promovieron nuevas rutas de incorporación a la vida académica.

Al comparar cómo ingresaron los académicos a las universidades en los años 1970 con respecto a las décadas más recientes, hay nuevas reglas de juego y nuevos perfiles. Los procesos de incorporación fueron diferentes según cada periodo. Como marco de referencia se consideran tres grandes periodos: de los años 1960 hasta 1982, o lo que se ha conocido como la "expansión no regulada" (Brunner, 1987), periodo de crecimiento en el que durante poco más de dos décadas tuvieron lugar cambios importantes en la estructura, normatividad y reglas de juego de las instituciones universitarias. El segundo periodo, abarca de 1983 a 1992. Representa el inicio de las políticas de evaluación y rendición de cuentas por parte de las universidades (De Vries, 2001; Rodríguez et al., 2003). Aquí pueden encontrarse nuevas formas de contratación de los académicos en las universidades. Finalmente, el tercer periodo, comprende de 1992 en adelante. Representa una nueva etapa de regulaciones en el sector académico con el ingreso de programas específicos tales como el SNI y el PRODEP.

Las variables consideradas para el análisis de esta dimensión fueron:

- el género de los académicos;

- el estado civil; 
- el grado máximo de estudios logrado por los académicos en el momento de la incorporación;

- el tipo de contrato con el que ingresaron;

- si participaban en PRODEP;

- y si participaban en el SNI.

La dimensión denominada "producción simbólica" se refiere a la relación entre los procesos de incorporación y los códigos de acceso que cada disciplina ha establecido a lo largo de su historia, y que con el tiempo devienen en códigos de comunicación; adquieren relevancia los premios y los reconocimientos en las trayectorias académicas y asignaciones son importantes para establecer procesos de diferenciación académica. Según Bourdieu (2005), la producción simbólica se asocia al prestigio y aporta elementos importantes para la construcción de la autonomía - incluso de la sobrevivencia - de un campo determinado.

La producción simbólica atiende la transmisión de los valores simbólicos, mediante prácticas derivadas de la membresía de una comunidad específica, la participación en redes académicas, de investigación, de producción del conocimiento, o bien, de vinculación con el ethos disciplinario (Vinck, 2014). Los agentes, al contar con múltiples referentes de identidad académica están expuestos a patrones de socialización según su campo de origen, de ahí que reconozcan sus tradiciones y prácticas simbólicas (Bourdieu, 2005).

Las variables de esta dimensión fueron:

- la facultad e institución de la licenciatura de origen, la instancia de realización del servicio social;

- los reconocimientos obtenidos en las trayectorias académicas;

- el tiempo transcurrido entre el egreso de la licenciatura;

- y el ingreso a la vida académica.

La dimensión sobre las dinámicas de "interacción social" que los académicos tuvieron en los años previos al ingreso a la vida académica, se refiere a la capacidad para establecer redes e integrarse a grupos específicos, lo cual varía según la lógica de cada disciplina y de los agentes académicos que construyen estrategias para incorporarse a las universidades.

La socialización previa de los académicos establece las formas en que experimentaron el mundo social cuando ellos aún eran estudiantes. La reproducción de ciertos comportamientos, valores, normas y hábitos van conformándose en el seno de una determinada disciplina, pero también en los patrones previos de convivencia y permanencia de los agentes en sus comunidades académicas de origen; es decir, durante la realización de la licenciatura y la entrada en contacto con el ethos de la academia por primera vez (Grediaga, 2000). Las variables en esta dimensión fueron:

- la construcción de expectativas hacia el empleo, la trayectoria previa y la formación profesional;

- las redes académicas previas;

- y la producción académica. 
La construcción estrategias para la incorporación de los académicos a las comunidades disciplinarias pasa por las dimensiones señaladas y varía según el contexto de cada disciplina. Aquí se empleó la comparación como método de investigación, al observar cómo los agentes se incorporan a la vida universitaria y qué diferencias disciplinarias se identifican.

En cada dimensión se hizo uso de la técnica de cluster $k$ means (del paquete estadístico SPSS) para obtener tipologías y derivar cuántas y cuáles estrategias de incorporación pueden identificarse en cada caso. También se recurrió al análisis factorial para graduar algunas variables. Después de cada tipología, se pusieron en correspondencia los tipos con las disciplinas para comparar las estrategias de incorporación. A partir de este procedimiento dio inicio el análisis y la discusión para revisar los supuestos de la hipótesis.

\section{ANÁLISIS Y DISCUSIÓN INICIAL}

Según la estadística institucional, la UV (2017) tiene una planta académica que asciende a 6.049 académicos distribuidos en tres funciones como docencia, investigación y difusión de la cultura. El $73 \%$ se dedica a la docencia y el resto a la investigación y difusión de la cultura.

En cuanto al tiempo de contratación, solo el $56,4 \%$ es personal de tiempo completo. La institución cuenta con $980(28 \%)$ académicos con el grado doctoral y $774(22 \%)$ con maestría en relación con la planta total. Con respecto a la pertenencia a los programas nacionales, la UV cuenta con 443 miembros en el sistema nacional de investigadores, $12 \%$ del total de los académicos de tiempo completo. Finalmente, en el PRODEP se registran 1.145 académicos, el 33\% de la planta de PTC. Las cifras anteriores indican una variabilidad en las condiciones de la planta académica, lo que implica la posibilidad de encontrar diversas formas de incorporarse a la institución.

\section{TIPOLOGÍA DE LA DIMENSIÓN DE ANÁLISIS: LAS POLÍTICAS EDUCATIVAS DIRIGIDAS AL SECTOR ACADÉMICO}

Para identificar las estrategias que construyeron los académicos en el proceso de incorporación, se procesaron las variables correspondientes con la técnica de cluster y se obtuvieron cinco tipos o perfiles (Cuadro 2).

Cada tipo resultó de una combinación específica que aportó elementos para la construcción de un perfil particular. El tipo 1 se constituyó por académicas con el grado de licenciatura, quienes concursaron por una o varias asignaturas; tenían la condición civil de casadas.

En el tipo 2, se agruparon nuevamente académicas, pero contaban con el grado de maestría al momento de la incorporación; eran solteras y obtuvieron un contrato parcial por la vía de la designación. En el tipo 3 se agruparon académicos, solteros, con la licenciatura como grado máximo y que ingresaron mediante un concurso por una o varias asignaturas. En los primeros tres tipos no hubo participación en el PRODEP ni en el SNI. 
El tipo 4 agrupó a académicos casados; con el grado doctoral al momento de la incorporación; concursaron por una plaza de tiempo completo y sí participaban en el PRODEP y el SNI. Finalmente, el tipo 5, cambió radicalmente. Eran académicos solteros al momento de la incorporación; no contaban con el título de licenciatura pero obtuvieron una plaza de tiempo completo por designación. En consecuencia no pertenecían a los programas federales mencionados. Las combinaciones resultantes aportaron más elementos al ponerse en correspondencia con las disciplinas.

Como se aprecia en la Tabla 1, las políticas dirigidas al sector de los académicos no generaron los mismos efectos entre las disciplinas. Las estrategias formuladas para incorporarse a la institución fueron diversas. El SNI y el PRODEP fueron recursos que solo una parte de la población estudiada integró a su perfil, pero en términos generales no tuvieron un rol definitivo como políticas de incorporación.

Con base en las distribuciones anteriores se identificaron dos estrategias claramente delimitadas. La primera estrategia consistió en el uso de los grados académicos y la participación en las políticas de PRODEP y SNI como parte de la formación de capital académico. Esta estrategia estuvo conformada básicamente por los casos que se agruparon en el tipo 2 y el tipo 4 . En ella tuvieron lugar dos orientaciones sobre el proceso de incorporación. La primera, incluyó a los académicos del género masculino que obtuvieron un grado doctoral, participaban en el SNI en el momento de su incorporación a la UV. Eran casados y obtuvieron un tiempo completo a través de un concurso de oposición. La segunda orientación integró a las académicas, quienes obtuvieron un grado de maestría, pero no participaban en el

Cuadro 2 - Perfiles de incorporación de los académicos en la dimensión "políticas educativas".

\begin{tabular}{|l|c|c|c|c|c|}
\hline \multirow{2}{*}{ Variable } & \multicolumn{5}{|c|}{ Tipos } \\
\cline { 2 - 6 } & Tipo 1 & Tipo 2 & Tipo 3 & Tipo 4 & Tipo 5 \\
\hline Género & Mujer & Mujer & Hombre & Hombre & Hombre \\
\hline Estado civil & Casada & Soltera & Soltero & Casado & Soltero \\
\hline $\begin{array}{l}\text { Grado de estudios } \\
\text { en el momento de } \\
\text { la incorporación }\end{array}$ & Licenciatura & Maestría & Licenciatura & Doctorado & $\begin{array}{c}\text { Sin } \\
\text { Licenciatura }\end{array}$ \\
\hline $\begin{array}{l}\text { Tipo de contrato } \\
\text { Procedimiento para } \\
\text { la obtención del } \\
\text { contrato }\end{array}$ & Asignatura & Asignatura & Asignatura & $\begin{array}{c}\text { Tiempo } \\
\text { completo }\end{array}$ & $\begin{array}{c}\text { Tiempo } \\
\text { completo }\end{array}$ \\
\hline $\begin{array}{l}\text { Participaba en el } \\
\text { PRODEP }\end{array}$ & No participó & No participó & No participó & Sí Participó & No participó \\
\hline $\begin{array}{l}\text { Participaba en el } \\
\text { SNI }\end{array}$ & No participó & No participó & No participó & Sí participó & No participó \\
\hline
\end{tabular}

Fuente: base de datos de búsqueda.

Elaboración propia. 
SNI y el PRODEP. Su condición civil era de solteras y su entrada a la universidad fue a través de un contrato parcial.

En la primera orientación hubo una fuerte presencia de académicos de las facultades de biología con un 50\% y matemáticas con un 20\%. Las dos disciplinas del cuadrante duro en el esquema de Becher se inclinaron por la incorporación de nuevos miembros cuando éstos tenían grados académicos de doctorado y cuando el SNI y el PRODEP eran parte de la certificación de sus trayectorias.

Con respecto a la segunda posibilidad, nuevamente biología destacó, esta vez por sus académicas con grado de maestría. Sin embargo, aparecieron otras disciplinas como letras españolas con un $60 \%$ de su planta académica en esta condición, mientras que sociología tuvo una representación del 40\% y matemáticas del 33\%. Las dos disciplinas del cuadrante duro y dos de las tres del cuadrante blando, ubicaron a sus académicas en esta estrategia. Cabe señalar que en este grupo solo letras tuvo mayor número de mujeres que hombres entre quienes participaron en este estudio.

La segunda estrategia obedeció más a las condiciones de la estructura universitaria que al efecto parcial de las políticas públicas. Se conformó por los tipos 1 y 5 , caracterizados por un conjunto de rasgos y acciones que definieron de otra manera el proceso de incorporación. Al igual que la estrategia anterior tuvo dos orientaciones específicas.

La primera orientación se formuló con académicos, quienes sin contar con el grado de licenciatura, obtuvieron un tiempo completo por designación de la facultad en la que actualmente laboran. Se trata de aquéllos que ingresaron a su

Tabla 1 - Perfiles de incorporación de los académicos en la dimensión "políticas educativas".

\begin{tabular}{|c|c|c|c|c|c|c|c|}
\hline \multirow{2}{*}{\multicolumn{2}{|c|}{ Facultad }} & \multicolumn{6}{|c|}{ Tipos } \\
\hline & & \multirow{2}{*}{$\begin{array}{c}\text { Tipo } 1 \\
1 \\
\end{array}$} & \multirow{2}{*}{$\begin{array}{c}\text { Tipo } 2 \\
6\end{array}$} & \multirow{2}{*}{$\begin{array}{c}\text { Tipo } 3 \\
-\end{array}$} & \multirow{2}{*}{$\begin{array}{c}\text { Tipo } 4 \\
7 \\
\end{array}$} & \multirow{2}{*}{$\begin{array}{c}\text { Tipo } 5 \\
- \\
\end{array}$} & \multirow{2}{*}{\begin{tabular}{c|} 
Tota \\
14
\end{tabular}} \\
\hline P. & NA & & & & & & \\
\hline B10log1a & $\%$ & 7 & 43 & - & 50 & - & 100 \\
\hline \multirow{2}{*}{ Matemáticas } & NA & 5 & 5 & 2 & 3 & - & 15 \\
\hline & $\%$ & 33 & 33 & 13 & 20 & - & 99 \\
\hline \multirow{2}{*}{ Pedagogía } & NA & 12 & 5 & 2 & - & 7 & 26 \\
\hline & $\%$ & 46 & 19 & 7 & - & 27 & 99 \\
\hline \multirow{2}{*}{ Sociología } & NA & 7 & 8 & - & - & 5 & 20 \\
\hline & $\%$ & 35 & 40 & - & - & 25 & 100 \\
\hline \multirow{2}{*}{ Letras } & NA & 4 & 12 & - & 1 & 3 & 20 \\
\hline & $\%$ & 20 & 60 & - & 5 & 15 & 100 \\
\hline \multirow{2}{*}{ Total } & $\mathrm{NA}$ & 29 & 36 & 4 & 11 & 15 & 95 \\
\hline & $\%$ & 30 & 38 & 4 & 12 & 16 & 100 \\
\hline
\end{tabular}

NA: número absoluto de participantes (total 95).

Fuente: base de datos de búsqueda.

Elaboración propia. 
disciplina antes de que las políticas regularan parte del proceso de incorporación. Pertenecen al primer periodo de análisis (de los años 1960 hasta 1982) establecido en esta dimensión. En aquél entonces era común obtener una plaza únicamente con la condición de pasante dadas las características de la masificación de la matrícula ya expuesta en la literatura correspondiente.

La segunda orientación incluyó a las académicas, quienes ingresaron a su disciplina únicamente con el grado de licenciatura y concursaron por una o varias asignaturas. Tenían la condición civil de casadas. La incorporación no se definió por algún efecto de las políticas públicas.

En la primera orientación de esta estrategia, se ubicaron prioritariamente los académicos de pedagogía con un $27 \%$ de los casos encuestados. Sociología tuvo una representación del $25 \%$ de los casos bajo estudio. En estas dos plantas académicas se encuentran los casos con mayor promedio de edad de la población estudiada, el cual asciende a 53 años. Es decir, en este grupo se encuentran quienes ingresaron antes del diseño de las políticas dirigidas al sector académico. En cierto modo, esta población representa un desafío para la UV al ser un grupo con más años impartiendo clases.

La segunda orientación de esta estrategia representó a los académicos de pedagogía, sociología y matemáticas, esta última con el tercer promedio de edad más alto del conjunto de académicos, el cual es de 51 años. Con la anterior distribución se observó que las disciplinas blandas usaron más los recursos propios de las dinámicas institucionales y menos aquellos que proceden de las políticas públicas. En el caso de matemáticas, el promedio de edad explica que un porcentaje de su planta académica se halle en esta orientación. A continuación se resume la estructura de las dos estrategias identificadas en la dimensión "políticas educativas" (Cuadro 3).

\section{TIPOLOGÍA DE LA DIMENSIÓN DE ANÁLISIS: LA “PRODUCCIÓN SIMBÓLICA”}

Para abordar esta dimensión y las estrategias que resultaron de ella, se recurrió nuevamente a la tipología a través del cluster. Se utilizaron las siete variables ya mencionadas en el apartado metodológico y con ellas se obtuvo una combinatoria que dio como resultado cuatro tipos o perfiles.

Como se aprecia en el Cuadro 4, los cuatro tipos resultantes reflejaron la importancia que tuvo el servicio social puesto que, al combinarse con el tiempo transcurrido entre el egreso de la licenciatura y la obtención del primer contrato, ${ }^{5}$ volvió más complejo el proceso de incorporación de los académicos.

El tipo 1 agrupa a quienes realizaron el servicio social en una dependencia relacionada directamente con su disciplina de origen cuando eran estudiantes,

5 Es importante señalar que en la dimensión "producción simbólica", se tomaron en cuenta variables relativas a reconocimientos en las trayectorias académicas, tales como obtención de premios en concursos académicos. La finalidad era explorar antecedentes relativos a la construcción de prestigio como rasgos que explicarían la entrada en redes académicas y, por consecuencia, la incorporación a la vida académica. Sin embargo, todas las variables referentes resultaron poco significativas. 
Cuadro 3 - Estrategias identificadas en la dimensión "políticas educativas".

\begin{tabular}{|c|c|c|}
\hline \multirow{2}{*}{$\begin{array}{l}\text { Estrategia } 1 \\
\text { Uso de grados académicos y } \\
\text { participación en las políticas } \\
\text { de SNI y PRODEP. }\end{array}$} & $\begin{array}{l}\text { Orientación } 1 \\
\text { Académicos que obtuvieron un grado doctoral, } \\
\text { participaban en el SNI en el momento de su } \\
\text { incorporación a la UV. Eran casados y obtuvieron un } \\
\text { tiempo completo a través de un concurso de oposición. }\end{array}$ & $\begin{array}{l}\text { Disciplinas } \\
\text { biología } \\
\text { matemáticas }\end{array}$ \\
\hline & $\begin{array}{l}\text { Orientación } 2 \\
\text { Académicas que obtuvieron un grado de maestría, } \\
\text { pero no participaban en el SNI y PRODEP. Su } \\
\text { condición civil era de solteras y su entrada a la } \\
\text { universidad fue a través de un contrato parcial. }\end{array}$ & $\begin{array}{l}\text { Disciplinas } \\
\text { biología } \\
\text { letras } \\
\text { sociología } \\
\text { matemáticas }\end{array}$ \\
\hline \multirow{2}{*}{$\begin{array}{l}\text { Estrategia } 2 \\
\text { Uso de recursos asociados } \\
\text { a las condiciones de la } \\
\text { estructura universitaria y a la } \\
\text { lógica de cada disciplina. }\end{array}$} & $\begin{array}{l}\text { Orientación } 1 \\
\text { Académicos, quienes sin contar con el grado de } \\
\text { licenciatura, obtuvieron un tiempo completo por } \\
\text { designación. Ingresaron a su disciplina antes de que las } \\
\text { políticas regularan parte del proceso de incorporación. }\end{array}$ & $\begin{array}{l}\text { Disciplinas } \\
\text { pedagogía } \\
\text { sociología }\end{array}$ \\
\hline & $\begin{array}{l}\text { Orientación } 2 \\
\text { Académicas quienes ingresaron a su disciplina } \\
\text { únicamente con el grado de licenciatura y } \\
\text { concursaron por una o varias asignaturas. Tenían la } \\
\text { condición civil de casadas. }\end{array}$ & $\begin{array}{l}\text { Disciplinas } \\
\text { pedagogía } \\
\text { sociología } \\
\text { matemáticas }\end{array}$ \\
\hline
\end{tabular}

Fuente: base de datos de búsqueda.

Elaboración propia.

Cuadro 4 - Perfiles de incorporación de los académicos en la dimensión "producción simbólica".

\begin{tabular}{|l|c|c|c|c|}
\hline \multirow{2}{*}{ Variable } & \multicolumn{4}{|c|}{ Tipos } \\
\cline { 2 - 5 } & Tipo 1 & Tipo 2 & Tipo 3 & Tipo 4 \\
\hline $\begin{array}{l}\text { Relación entre el servicio } \\
\text { social y la disciplina de origen }\end{array}$ & Sí & No & No \\
\hline $\begin{array}{l}\text { Relación entre el servicio } \\
\text { social y el primer contrato }\end{array}$ & No decisivo & Decisivo & No decisivo & Decisivo \\
\hline $\begin{array}{l}\text { Relección de la dependencia } \\
\text { para realizar el servicio social }\end{array}$ & $\begin{array}{c}\text { Promovido por } \\
\text { la dependencia } \\
\text { del servicio } \\
\text { social }\end{array}$ & $\begin{array}{c}\text { Promovido } \\
\text { de manera } \\
\text { individual }\end{array}$ & $\begin{array}{c}\text { Promovido por } \\
\text { la facultad }\end{array}$ & $\begin{array}{c}\text { Promovido por } \\
\text { la dependencia } \\
\text { del servicio } \\
\text { social }\end{array}$ \\
\hline $\begin{array}{l}\text { Tiempo entre el egreso de } \\
\text { la licenciatura y el primer } \\
\text { contrato }\end{array}$ & $\begin{array}{c}\text { Mismo año de } \\
\text { egreso }\end{array}$ & $\begin{array}{c}\text { Cinco años } \\
\text { posteriores al } \\
\text { egreso }\end{array}$ & $\begin{array}{c}\text { Ya tenía un } \\
\text { contrato al } \\
\text { egreso }\end{array}$ & $\begin{array}{c}\text { Entre dos y } \\
\text { cinco años }\end{array}$ \\
\hline $\begin{array}{l}\text { Tiempo entre el egreso y el } \\
\text { primer contrato en la UV }\end{array}$ & $\begin{array}{c}\text { Más de } \\
\text { quince años } \\
\text { posteriores al } \\
\text { egreso }\end{array}$ & $\begin{array}{c}\text { Más de 15 años } \\
\text { posteriores al } \\
\text { egreso }\end{array}$ & No aplica & $\begin{array}{c}\text { Entre dos y } \\
\text { cinco años }\end{array}$ \\
\hline Egresó de la UV & No & No & Sí & Sí \\
\hline $\begin{array}{l}\text { Realizó el servicio social en } \\
\text { la UV }\end{array}$ & No & No & No & No \\
\hline
\end{tabular}

Fuente: base de datos de búsqueda.

Elaboración propia. 
aunque no se trataba de una instancia de la UV. El servicio social fue promovido por la dependencia que lo ofrecía. Estos académicos obtuvieron su primer contrato en el mismo año en que egresaron de la licenciatura; sin embargo, pasaron quince años para concretar su entrada en la UV. No egresaron de la UV y el servicio social no fue decisivo en el primer contrato.

E1 tipo 2, agrupó a quienes no hicieron el servicio social en una instancia relativa a su disciplina, pero éste contribuyó a la obtención de su primer contrato, si bien éste se dio cinco años después del egreso de la licenciatura. Estos académicos buscaron el servicio por su propia cuenta y también tardaron quince años en incorporarse a la UV, dado que no egresaron de ella.

El tipo 3, realizó el servicio social en una dependencia relativa a la disciplina, pero ya contaban con un contrato en esta fase de su vida estudiantil. Sin duda se trata de aquellos académicos que en la dimensión anterior obtuvieron su primer contrato en la UV sin contar con el título de licenciatura. La propia facultad promovió el servicio social. Egresaron de la UV, aunque el servicio lo hicieron en otra dependencia.

Finalmente, el tipo 4, identificó a quienes el servicio social aportó a la obtención de su primer contrato aunque no se relacionara con su disciplina de origen. Fue promovido por la dependencia donde se llevó a cabo, y los académicos tardaron entre dos y cinco años para conseguir su primer contrato y, posteriormente, ingresar en ese mismo tiempo a la UV. Egresaron de esta casa de estudios.

$\mathrm{Al}$ poner en correspondencia los datos de la Tabla 2 con las disciplinas bajo estudio encontramos las siguientes distribuciones.

Tabla 2 - Perfiles de incorporación de los académicos en la dimensión "producción simbólica".

\begin{tabular}{l|c|c|c|c|c|c}
\hline \multirow{2}{*}{ Facultad } & \multicolumn{6}{c}{ Tipos } \\
\cline { 3 - 7 } \multicolumn{2}{c|}{} & Tipo 1 & Tipo 2 & Tipo 3 & Tipo 4 & Total \\
\hline \multirow{2}{*}{ Biología } & NA & 1 & 6 & - & 7 & 14 \\
\cline { 2 - 7 } & $\%$ & 7 & 43 & - & 50 & 100 \\
\hline \multirow{3}{*}{ Matemáticas } & NA & 3 & 3 & - & 9 & 15 \\
\cline { 2 - 7 } & $\%$ & 20 & 20 & - & 60 & 100 \\
\hline \multirow{3}{*}{ Pedagogía } & NA & 6 & - & 9 & 11 & 26 \\
\cline { 2 - 7 } & $\%$ & 23 & - & 35 & 42 & 100 \\
\hline \multirow{3}{*}{ Sociología } & NA & 5 & 2 & 2 & 11 & 20 \\
\cline { 2 - 7 } & $\%$ & 25 & 10 & 10 & 55 & 100 \\
\hline \multirow{3}{*}{ Total } & NA & 2 & 3 & 6 & 9 & 20 \\
\cline { 2 - 7 } & $\%$ & 10 & 15 & 30 & 45 & 100 \\
\hline & NA & 17 & 13 & 18 & 47 & 95 \\
\hline
\end{tabular}

NA: número absoluto de participantes (total 95).

Fuente: base de datos de búsqueda.

Elaboración propia. 
Siguiendo el Cuadro 5, los tipos reflejan en grado mayor o menor la influencia de las disciplinas y sus estructuras simbólicas mediadas por la forma en que producen prácticas y códigos específicos para crear escenarios de incorporación de sus nuevos miembros. Se pueden identificar dos grandes estrategias construidas en esta dimensión. La primera estrategia puede describirse como una producción simbólica altamente estructurada, donde el servicio social jugó un papel importante. Esta estrategia se conformó por los tipos 3 y 4 . Como ocurrió con la dimensión "políticas públicas", aquí también se formularon dos orientaciones principales.

La primera orientación implica a los académicos que egresaron de la misma disciplina en la que actualmente laboran. Hicieron el servicio social en instancias que al tener una relación directa con la disciplina eran espacios formativos de personal académico para adquirir condiciones favorables para su reclutamiento en la institución. Es el único grupo donde la facultad promovió el servicio social en este tipo de dependencias. Se trata además de académicos que sin haber egresado ya contaban con un contrato.

La segunda orientación presenta a los académicos que aun cuando no realizaron el servicio social en una dependencia relacionada directamente con la disciplina de origen, reportaron que el servicio social fue decisivo para encontrar el primer empleo. Estos académicos tardaron entre dos y cinco años en obtener el contrato en la UV y fueron egresados de la misma.

En la primera orientación aparecen pedagogía y letras; es decir, dos disciplinas del cuadrante blando. Pedagogía concentró el 35\% de sus académicos aquí, mientras que letras un 30\%. La influencia de la disciplina en el proceso de incorporación se muestra porque a través del servicio social se promueve a los académicos en quienes

Cuadro 5 - Estrategias identificadas en la dimensión "producción simbólica".

\begin{tabular}{|l|l|c|}
\hline \multirow{5}{*}{$\begin{array}{l}\text { Estrategia 1 } \\
\text { Producción simbólica } \\
\text { altamente estructurada. }\end{array}$} & $\begin{array}{l}\text { Orientación 1 } \\
\text { Académicos que egresaron de la misma facultad } \\
\text { en la que actualmente laboran. Hicieron el servicio } \\
\text { social en instancias relacionadas con la disciplina. } \\
\text { Ya contaban con un contrato en la facultad. }\end{array}$ & $\begin{array}{c}\text { Disciplinas } \\
\text { pedagogía } \\
\text { letras }\end{array}$ \\
\cline { 2 - 4 } & $\begin{array}{l}\text { Orientación 2 } \\
\text { Académicos que egresaron de la misma facultad } \\
\text { en la que laboran actualmente. El servicio social } \\
\text { no se realizó en una entidad relacionada con } \\
\text { la disciplina, pero fue decisivo para obtener el } \\
\text { primer contrato. Tardaron entre dos y cinco años } \\
\text { para ingresar a la UV. }\end{array}$ & $\begin{array}{c}\text { Disciplinas } \\
\text { biología } \\
\text { sociología } \\
\text { matemáticas }\end{array}$ \\
\hline $\begin{array}{l}\text { Estrategia 2 } \\
\text { Producción simbólica poco } \\
\text { estructurada. }\end{array}$ & $\begin{array}{l}\text { Orientación 1 } \\
\text { Académicos cuya estrategia no se formuló a la luz } \\
\text { de la facultad donde laboran, sino de la disciplina } \\
\text { en cuestión. El servicio social tuvo relación con } \\
\text { la disciplina de origen. Tardaron quince años en } \\
\text { incorporarse a la UV lo cual permitió construir } \\
\text { una trayectoria acorde a las políticas públicas. }\end{array}$ & $\begin{array}{c}\text { Disciplinas } \\
\text { biología } \\
\text { sociología } \\
\text { matemáticas }\end{array}$ \\
\hline
\end{tabular}

Fuente: base de datos de búsqueda.

Elaboración propia. 
está previsto el ingreso a la comunidad académica. Es probable que este fuera un paso previo para quienes obtuvieron el contrato en la UV antes de egresar como estudiantes de licenciatura.

Con respecto a la segunda orientación, destacaron las disciplinas de matemáticas, sociología y biología, con más de la mitad de sus académicos. Es decir, aquí se ubicaron de forma predominante las disciplinas del cuadrante duro. Este grupo recibió una influencia estructural un tanto diferente al de la primera orientación, pues mientras en aquél la misma facultad se encargó de encauzar a aquellos agentes que posteriormente reclutó, aquí tuvo más relevancia el servicio social en la obtención del primer contrato.

La segunda estrategia fue denominada como una producción simbólica poco estructurada. Se conformó por los tipos 1 y 2, para mostrar cómo los agentes no siempre definen sus acciones a la luz de las disciplinas. En esta estrategia solo hubo una orientación general, pues los dos tipos son similares. Se identificaron aquí los académicos cuyos recursos no procedieron de la facultad donde se incorporaron a la vida académica. Con excepción de hecho de que el servicio social tuviera relación con la disciplina de origen, para estos agentes el servicio social no fue decisivo para conseguir su contrato en la UV, ni fue promovido por la facultad de origen. Esto es una consecuencia lógica porque no fueron egresados de la UV.

Las disciplinas con mayor representación en esta orientación fueron (sumando los porcentajes de ambos tipos) biología y matemáticas, ambas del cuadrante duro de Becher. En estas dos disciplinas hubo un menor nivel de endogamia, en el sentido de que la incorporación de los nuevos miembros fue con académicos no egresados de la UV, quienes tardaron más de quince años en ingresar a sus respectivas comunidades disciplinarias (Cuadro 5).

\section{TIPOLOGÍA DE LA DIMENSIÓN DE ANÁLISIS: LOS PROCESOS DE “SOCIALIZACIÓN Y LA CONSTRUCCIÓN DE EXPECTATIVAS" HACIA LA DISCIPLINA DE ORIGEN}

Esta dimensión recuperó los procesos de interacción social que dieron forma a las trayectorias de los académicos en su etapa de estudiantes. Como ya se estableció, se procesaron nuevamente siete variables cuya combinación arrojó tres tipos al usar la técnica de Cluster. También se usó la técnica de análisis factorial para graduar la variable "Nivel de expectativas de los académicos hacia su disciplina de origen", la cual se integró a la construcción de la tipología (Cuadro 6).

En el tipo 1, los académicos tenían expectativas medias para trabajar en la profesión académica orientada hacia la disciplina de origen. Pero no se observa una socialización como aspecto clave para incorporarse. Aunque asistieron a congresos, no participaron durante la licenciatura en proyectos de investigación; sin embargo, los distingue que tuvieron docencia en el nivel superior al menos durante cinco años. No realizaron actividades de investigación como profesionales ni fueron promovidos en su empleo por colegas de la institución donde actualmente laboran.

En el tipo 2, los académicos reportaron un nivel bajo en las expectativas sociales y profesionales hacia su disciplina de origen. No tenían claridad sobre la profesión 
académica como actividad laboral. No participaron en proyectos de investigación durante su trayectoria estudiantil. En su trayectoria profesional no tenían experiencia en investigación ni docencia previa a su ingreso a la UV. Si bien asistieron a congresos fue en calidad de oyentes. El factor decisivo para obtener el empleo fue, sin duda, mantenerse dentro de la red de socialización de su facultad de origen.

El tipo 3 agrupó a los académicos que estratégicamente conciliaron mejor los recursos propios del campo universitario y construyeron un perfil más académico para incorporarse a la UV. Estos agentes tenían socialización previa en la licenciatura dentro del campo de la investigación y parte de su trayectoria fue integrada a las redes académicas. Las expectativas hacia el trabajo académico relativo a su disciplina de origen eran altas. Asistieron a congresos en su calidad de estudiantes y lo hicieron con una participación como ponentes. Tuvieron una trayectoria de investigación ya en su vida profesional y antecedentes de docencia en el nivel superior. Fueron las propias redes de su facultad de origen las que promovieron el ingreso a la comunidad disciplinaria (Tabla 3).

La relación entre las disciplinas y los tipos resultantes de esta dimensión arrojó procesos y patrones diferentes en las trayectorias de los académicos y la exposición a una interacción social muy variada. Se observa cómo las disciplinas fueron guiando dicha interacción entre los académicos desde su condición de estudiantes. Las relaciones presentadas dan pauta para la identificación de dos estrategias nuevas.

La primera estrategia fue la incorporación al trabajo académico como resultado de una socialización académica sin antecedentes en actividades de investigación. Está conformada por los tipos 1 y 2, aunque una porción de los académicos tuvo

Cuadro 6 - Perfiles de incorporación de los académicos en la dimensión "procesos de socialización".

\begin{tabular}{|l|c|c|c|}
\hline \multirow{2}{*}{ Variable } & \multicolumn{3}{|c|}{ Tipos } \\
\cline { 2 - 4 } & Tipo 1 & Tipo 2 & Tipo 3 \\
\hline $\begin{array}{l}\text { Nivel de expectativas de los académicos } \\
\text { hacia su disciplina de origen }\end{array}$ & Medio & Bajo & Alto \\
\hline $\begin{array}{l}\text { Participación en proyectos de } \\
\text { investigación durante la licenciatura }\end{array}$ & No & No & Sí \\
\hline $\begin{array}{l}\text { Asistencia a congresos académicos } \\
\text { durante la licenciatura }\end{array}$ & Sí & Sí & Sí \\
\hline $\begin{array}{l}\text { Tipo de participación en congresos } \\
\text { académicos }\end{array}$ & Asistente & Asistente & Ponente \\
\hline Promocionaron el primer empleo & $\begin{array}{c}\text { Las redes } \\
\text { familiares }\end{array}$ & Las disciplinas & Las disciplinas \\
\hline Docencia en el nivel superior & $\begin{array}{c}\text { Al menos cinco } \\
\text { años }\end{array}$ & No & $\begin{array}{c}\text { Más de cinco } \\
\text { años }\end{array}$ \\
\hline Actividades de investigación & No & No & Sí \\
\hline
\end{tabular}

Fuente: base de datos de búsqueda.

Elaboración propia. 
antecedentes de docencia de al menos cinco años mientras que otra porción no registró docencia como actividad previa. Resulta interesante que las expectativas construidas por este grupo en general van de medias a bajas; es decir, no tenían una fuerte proyección para pertenecer a las comunidades disciplinarias en las que ahora se desempeñan.

La segunda estrategia se conformó únicamente por el tipo 3. Se distingue porque los antecedentes de socialización transcurrieron en actividades de investigación; su experiencia docente previa fue de más de cinco años. Son los académicos que tenían mayor claridad sobre la profesión académica relativa a su disciplina de origen, independientemente del establecimiento en el que ellos pudieran ubicarse. Este grupo participó con ponencias en congresos de investigación cuando estudiaban la licenciatura. Fueron promovidos por académicos de las disciplinas en las que actualmente laboran.

En la primera estrategia, sumando los porcentajes de los tipos 1 y 2 , se tiene que las tres disciplinas con mayor representación fueron las blandas: pedagogía con un 70,7\% de los casos, letras y sociología 65\% y 60\%, respectivamente. La ubicación de casi dos terceras partes de la población total de las disciplinas blandas es consistente con las distribuciones vistas en las dimensiones anteriores.

Por su parte, las disciplinas duras estuvieron más representadas en la segunda estrategia. Matemáticas ubicó al $67 \%$ de sus agentes sociales, mientras que biología a

Tabla 3 - Perfiles de incorporación de los académicos en la dimensión "procesos de socialización".

\begin{tabular}{l|c|c|c|c|c}
\hline \multirow{2}{*}{ Facultad } & \multicolumn{5}{c}{ Tipos } \\
\cline { 3 - 6 } \multicolumn{2}{c}{ Biología } & Tipo 1 & Tipo 2 & Tipo 3 & Total \\
\cline { 2 - 6 } & $\%$ & 2 & 3 & 9 & 14 \\
\hline \multirow{3}{*}{ Matemáticas } & NA & 4 & 21 & 64 & 99 \\
\cline { 2 - 6 } & $\%$ & 27 & 1 & 10 & 15 \\
\hline \multirow{3}{*}{ Pedagogía } & NA & 12 & 6 & 67 & 100 \\
\cline { 2 - 6 } & $\%$ & 46 & 30,7 & 6 & 26 \\
\hline \multirow{3}{*}{ Sociología } & NA & 4 & 8 & 8 & 99,7 \\
\cline { 2 - 6 } & $\%$ & 20 & 40 & 40 & 20 \\
\hline \multirow{3}{*}{ Letras } & NA & 5 & 8 & 7 & 20 \\
\cline { 2 - 6 } & $\%$ & 25 & 40 & 35 & 100 \\
\hline \multirow{2}{*}{ Total } & NA & 27 & 28 & 40 & 95 \\
\cline { 2 - 6 } & $\%$ & 28,4 & 29,4 & 42 & 99,8 \\
\hline
\end{tabular}

Fuente: base de datos de búsqueda.

Elaboración propia. 
un 64\%. En estas disciplinas dos tercios de la población entraron en redes académicas como resultado de una socialización que pasó por diferentes vías. La socialización desembocó también en un proceso de incorporación exitoso.

\section{CONCLUSIONES}

Como ha podido constatarse, la variabilidad producida al interior de cada dimensión de análisis se mantuvo dentro de los cauces de las disciplinas; es decir, éstas fueron las que permitieron ordenar las variables que intervinieron en la construcción de trayectorias académicas y perfiles que dieron pauta a los procesos de incorporación.

La apuesta inicial en la hipótesis fue que los procesos de incorporación de los académicos a las disciplinas derivan de una diversidad de racionalidades que dan origen a situaciones estratégicas que los agentes sociales ponen en juego. La construcción de estrategias implica los recursos internos a la institución y a las disciplinas así como a los recursos propios de las políticas públicas.

Puede concluirse que las relaciones planteadas en la hipótesis se cumplen; sin embargo, posterior al estudio, surgieron nuevos planteamientos para recuperarse en las conclusiones del artículo. En cada dimensión de análisis se observó que las disciplinas respondieron de una forma específica a las regulaciones de las políticas públicas, tanto como a la producción simbólica, y a la socialización y construcción de expectativas. Pero el eje que articuló tal cuestión fue su ubicación en el cuadrante de las duras o de las blandas. Esto aportó un marco explicativo del comportamiento de las disciplinas y los académicos.

En la primera dimensión, las disciplinas del cuadrante duro usaron más los recursos de las políticas públicas. Es más frecuente encontrar que los académicos responden a una racionalidad donde contar con grados académicos y pertenecer a los programas federales como el SNI y el PRODEP forman parte de la construcción de perfiles adecuados en el proceso de incorporación (Estrategia 1, primera orientación). El único factor que rompe con esta lógica es el de la edad.

De esta forma, en matemáticas y biología, las estrategias construidas por los académicos obedecieron a esa racionalidad externa que representan las disposiciones federales, las cuales en su diseño suelen coincidir con los criterios "duros" para la construcción de conocimiento científico, algo que puede estar a menor alcance de otras disciplinas.

Un aspecto que merece la atención se refiere a la relación entre las políticas públicas y las cuestiones de género, la cual si bien no formaba parte central del estudio, señala un fenómeno de relevancia actual en la composición de las plantas académicas. Mientras los académicos tenían grados doctorales en el momento de la incorporación, las académicas se asociaron más a los grados de maestría (Estrategia 1 , segunda orientación). Esto ya ha sido señalado en la literatura correspondiente en el caso de otras instituciones estudiadas. Gil Antón, Padilla y Galáz (2016) ${ }^{6}$

6 En el ya citado trabajo coordinado por Galáz y otros (2012). 
señalaron que en el 2010, las mujeres tenían mayor relación con el grado de maestría que con el grado doctoral. ${ }^{7}$ De esta forma, en biología se tiene que hombres y mujeres hicieron uso de los grados académicos si bien de manera diferenciada en el proceso de incorporación, mientras que en matemáticas, debido a la distribución de su planta académica, la proporción de mujeres con el grado de maestría fue menor.

Por su parte, las disciplinas del cuadrante blando, también hicieron uso parcial de los grados académicos, pero destaca el grado de maestría. Letras españolas y sociología ubicaron porcentajes significativos en esta condición. Con sociología se rompe relativamente la relación que se venía identificando con respecto al género y las políticas públicas, pues en su distribución hay mayoría de hombres. Puede concluirse que en el caso de las disciplinas blandas es menor el uso de las políticas públicas como recursos para iniciar el proceso de incorporación a la vida académica; sin embargo no deja de estar presente.

Las disciplinas blandas, fueron las únicas en las que un porcentaje de sus académicos iniciaron su vida académica sin haber obtenido el grado de licenciatura. Particularmente, pedagogía y sociología tuvieron más de una cuarta parte de su población en este estudio bajo esa condición (Estrategia 2, primera orientación). Al ser las plantas con mayor edad, hicieron mayor uso de los recursos de la institución que de los propios de las políticas públicas, con lo cual se concluye que en estas disciplinas el proceso de incorporación puede ser más laxo que en el de las duras. De hecho, aún en el momento presente, en las disciplinas blandas todavía es posible ingresar a la vida académica únicamente con el grado de licenciatura (Estrategia 2, segunda orientación), cuestión que en las disciplinas duras prácticamente ha desaparecido.

Con respecto a la dimensión "producción simbólica", nuevamente se observa que las racionalidades construidas por las disciplinas y los académicos varían en función de su pertenencia a uno u otro cuadrante. Aquí están los primeros atisbos de la socialización condensada en el servicio social como momento clave para el inicio de la vida académica en la UV. En esta dimensión está en juego el grado de estructuración y regulación que producen las disciplinas.

En el caso de las disciplinas blandas, la relación se observa con un alto grado de estructuración con la producción simbólica. Pedagogía y letras recibieron una regulación a través de dos fuentes principales, el servicio social y la propia facultad en la que actualmente laboran los académicos (Estrategia 1, primera orientación). Esto se concluye porque fue la misma facultad la que brindó un contrato sin haber egresado, al mismo tiempo quien dirigió a los académicos a los espacios de servicio social convenientes para el logro de ese contrato.

Por su parte, las disciplinas duras, tuvieron mayor tendencia hacia las trayectorias de académicos donde el servicio social funcionó más para obtener el primer contrato, no así para lograr su incorporación en la disciplina donde actualmente laboran (Estrategia 1, segunda orientación). Es definitivo que en ambas orientaciones el servicio social fue decisivo.

7 Vale la pena insistir en que se trataba de una tendencia más no de la generalidad. De hecho, en el 2016 la proporción entre hombres y mujeres con el grado doctoral ha evolucionado en favor de las académicas. 
Las disciplinas duras tuvieron menor regulación de parte de las facultades donde actualmente laboran, en parte porque los académicos no fueron estudiantes egresados de esas facultades. Biología y matemáticas reclutaron personal con trayectorias más elaboradas (Estrategia 2). La regulación que recibieron los académicos no provino de las facultades sino de las disciplinas en cuestión, de ahí que sus perfiles fueran estratégicamente exitosos, pues lograron construirlos al margen de sus espacios laborales actuales, particularmente con una trayectoria de docencia de más de quince años.

Finalmente, en lo referente a la dimensión "socialización y construcción de expectativas", se tiene que la relación entre las disciplinas y las estrategias de incorporación reveló nuevas rutas de incorporación. Esta vez fue el grado de participación en actividades de investigación previas las que distinguieron a las disciplinas duras de las blandas.

Las disciplinas blandas tienen casi dos tercios de sus académicos como aquellos quienes su tiempo de socialización previa no pasó por actividades de investigación. La socialización se desplegó en la docencia, aunque de manera relativa. Puesto que estos académicos o bien no tuvieron antecedentes de docencia en alguna otra institución antes de ingresar a su disciplina actual, o bien, sólo tuvieron un periodo corto de cinco años. Las expectativas que ellos tenían hacia la vida académica en la disciplina donde actualmente laboran iban de medias a bajas. Llama la atención el papel que jugaron las familias para la obtención del primer empleo en la trayectoria de los académicos (Estrategia 1). Esto se explica en parte porque algunas profesiones como pedagogía, suelen tener referencias antecedentes en casa. Algunos autores han señalado que el peso de las familias es determinante en las elecciones que hacen los hijos tanto en su trayectoria laboral como social en general. La profesión docente es fuertemente endogámica, tiende a reproducirse en el interior de las configuraciones familiares (Tenti, 2007). Si bien hay una proporción de académicos que escapa a esta condición, es claro que las disciplinas blandas las actividades de investigación están menos presentes y no representan espacios de socialización previa.

Por su parte, los académicos de las disciplinas duras tuvieron mayor inclinación a involucrarse en actividades de investigación cuando eran estudiantes $\mathrm{y}$, posteriormente, en su vida profesional previa a la incorporación a la disciplina actual. Esto concuerda con el hecho de que su docencia se desplegó por más de cinco años y las expectativas construidas hacia el trabajo académico se registraron en un nivel alto (Estrategia 2). Matemáticas y biología reportaron dos tercios de su población en esta condición.

El proceso de incorporación de los académicos a las instituciones y a las disciplinas en particular es altamente complejo y requiere de la intervención de diversas dimensiones de análisis para contar con referentes empíricos y analíticos capaces de abordar la diversidad de estrategias que construyen los académicos en el marco de sus disciplinas de referencia. Parte de su complejidad está asociada a otros fenómenos que actualmente están presentes en las instituciones universitarias, tales como la jubilación y el relevo generacional, que en cierta añaden nuevos elementos y diversifican aún más las fórmulas para ingresar a las instituciones. 
Es claro que en el panorama actual, las políticas públicas han regulado parcialmente los perfiles académicos, pero aún guardan distancia con respecto a las formas en que las instituciones construyen y ponen en juego sus rutas para incorporar a los académicos a las comunidades disciplinarias, que en parte tiene que ver con las configuraciones de autonomía que caracterizan a las instituciones de producción y transmisión de la cultura y el conocimiento.

\section{REFERENCIAS}

Altbach, P.; Finkelstein, M. The academic profession. Portraits of fourteen countries. Princeton, NJ: The Carnegie Foundation for the Advancement of Teaching, 1997.

Becher, T. Tribus y territorios académicos. La indagación intelectual y las culturas de las disciplinas. Barcelona: Gedisa, 1997.

Bourdieu, P. Respuestas. Por una antropología reflexiva. México: Grijalbo, 1995. . Homo academicus. México: Siglo XXI, 2005.

Brunner, J. Universidad y sociedad en América Latina. Ciudad de México: ANUIES; UAM, 1987.

Clark, B. El sistema de educación superior. México: Nueva Imagen; UAM, 1991.

Crozier, F.; Friedberg, E. El actor y el sistema. México: Alianza Editorial, 1990.

De VRIEs, W. El exorcismo de demonios y ángeles. Los efectos de políticas públicas sobre el trabajo académico. México: Universidad Autónoma de Aguascalientes, 2001.

Galáz, J.; Arimoto, A.; Teichler, U.; Brennan, J. (Eds.). Biographies and careers throughout academic life. The changing academy - the changing academic profession in international comparative perspective. Switzerland: Springer, 2016.

Galáz, J.; Gil Antón, M.; Padilla, L.; Sevilla, J.; Arcos, J.; Martínez, J. La reconfiguración de la profesión académica en México. México: Universidad Autónoma de Sinaloa; Universidad Autónoma de Baja California, 2012.

Gil Antón, M.; Padilla, 1.; Galáz, J. The mexican academic profession between centuries: who are? In: Galáz, J.; Arimoto, A.; Teichler, U.; Brennan, J. (Eds.). Biographies and careers throughout academic life. The changing academy - the changing academic profession in international comparative perspective. Switzerland: Springer Nature, 2016. p. 67-88.

Gil Antón, M. Los rasgos de la diversidad: un estudio sobre los académicos mexicanos. México: UAM, 1994. Contribución Equipo Interinstitucional de Investigadores sobre los Académicos Mexicanos.

Grediaga, R. Profesión académica: disciplinas y organizaciones. Procesos de socialización y sus efectos en las actividades y resultados de los académicos mexicanos. Ciudad de México: ANUIES, 2000.

Grediaga, R.; Rodríguez, J.; Padilla, L. Politicas públicas y cambios en la profesión académica en México en la última década. Ciudad de México: ANUIES; UAM, 2004. 
Rodríguez, J.; Grediaga, R.; Padilla, L.; Campos, M. Variaciones de una misma orientación general. Las políticas públicas hacia la educación superior en Argentina, Brasil, Chile, México y Venezuela. Revista de la Educación Superior, Ciudad de México: ANUIES, v. 4, n. 128, p. 29-58, 2003.

Tenti, E. La condición docente. Buenos Aires: Siglo XXI, 2007.

UV - Universidad Veracruzana. Series históricas 2008-2017. México: UV, 2017. Disponible en: <https://www.uv.mx/informacion-estadistica/files/2018/01/SeriesHistoricas-2017_2018-Final.pdf>. Acceso en: 4 jul. 2018.

Vinck, D. Ciencias y sociedad. Sociología del trabajo científico. Barcelona: Gedisa, 2014.

\section{SOBRE LOS AUTORES}

Eréndira García García es maestra en investigación educativa por la Universidad Veracruzana (México). Profesora de la misma institución. E-mail: erendira_garcia90@hotmail.com

José Luis SuÁrez Domínguez es doctor en sociología por la Universidad Autónoma Metropolitana (México). Profesor de la Universidad Veracruzana (México).

E-mail:1suarez@uv.mx

Recibido el $1^{\circ}$ de septiembre de 2017 Aprobado el 22 de febrero de 2018 